Article

\title{
Thermal Kinetics of a Lignin-Based Flame Retardant
}

\author{
Xiaoxuan Liang, Qixiang Hu, Xu Wang, Liang Li, Yuguo Dong, Chang Sun, Chengjuan Hu \\ and Xiaoli $\mathrm{Gu}$ *(1) \\ Co-Innovation Center for Efficient Processing and Utilization of Forest Products, College of Chemical \\ Engineering, Nanjing Forestry University, Nanjing 210037, China; xiaoxuanliang1998@outlook.com (X.L.); \\ hu_qixiang@outlook.com (Q.H.); xuWang2020@outlook.com (X.W.); liliang250035@gmail.com (L.L.); \\ dongyuguo99@outlook.com (Y.D.); 18851942018sc@sina.com (C.S.); hcj180203406@hotmail.com (C.H.) \\ * Correspondence: guxiaoli@njfu.edu.cn; Tel.: +86-25-8542-7624
}

Received: 30 August 2020; Accepted: 15 September 2020; Published: 17 September 2020

\begin{abstract}
In order to improve the thermal property of epoxy resin (EP), a lignin-based flame retardant was prepared. Focusing on the lignin-based flame retardant, this paper investigates its pyrolysis behavior and kinetics via a thermogravimetric analyzer coupled with Fourier transform infrared spectrometry (TG-FTIR). Based on the FTIR result, which showed a peak at $1222 \mathrm{~cm}^{-1}$, it was assigned a syringyl structure. Its absorption peak intensity was enhanced and this meant that the phenolization of the lignin was successful. Thermogravimetry/derivative thermogravimetry (TG/DTG) results showed that the carbon residues of F-lignin and F-lignin@APP were reduced to 33.5\% and 37.5\%, respectively. In addition, the maximum decomposition rate of F-lignin@APP20/EP is 11.8\%/min, which is $8 \% / \mathrm{min}$ and $4.7 \% / \mathrm{min}$ lower than for EP and Al-lignin, respectively. The char residue of F-lignin@APP20/EP is 32.5\%, which is much higher than for EP. Lower decomposition rate and higher char residue indicate the improvement of thermal stability of EP by F-lignin@APP. Moreover, the kinetics of Al-lignin20/EP and F-lignin@APP20/EP were conducted by two kinetic methods: Flynn-Wall-Ozawa (FWO) and Kissinger-Akahira-Sunose (KAS). It was concluded that the pyrolysis process of Al-lignin 20/EP and F-lignin@APP 20/EP could be divided into three stages, while the value and growth rate of the activation energy of F-lignin@APP 20/EP were much higher than that of Al-lignin 20/EP in stage III.
\end{abstract}

Keywords: lignin; flame retardant; kinetic analysis

\section{Introduction}

Epoxy resin (EP) is widely applied in daily life, such as in insulating materials used in electronic devices, semiconductor device plastic packaging, automotive adhesives, building decoration, etc. [1-4]. There are many types of EP, among which the largest output and the most complete variety are bisphenol A epoxy resins. These account for more than $90 \%$ of the EP market share [5]. Although EP has excellent properties, such as easy curing and work, good mechanical properties, good heat resistance and solvent resistance, its poor flammability has seriously affected its use in life. The limiting oxygen index (LOI) of epoxy resin is about $20 \%$, which means it will burn quickly after being ignited [6]. Therefore, it is very important to improve the flame retardant performance of epoxy resin.

A lot of research has been reported on the thermal behavior of epoxy resins [7-9], and a variety of methods have been proposed to improve its thermal performance, including the incorporation of aromatic rings and fire-retarding additives into the epoxy crosslinked network [10-12] or the copolymerization of epoxy resins with reactive fire retardants [13]. The thermal property and flammability of epoxy resins are strongly influenced by the chemical structure of the resins, the nature of the curing agents used and the crosslink density of the final products [9]. The aromatic epoxy resins exhibit a higher thermal stability than the aliphatic one, generally [7]. The influence of the chemical 
structure of an epoxy resin on its thermal stability has been reported [8]. In general, the thermal stability of a particular epoxy resin enhances with the increase of its crosslink density [9].

As the most abundant polymer in nature other than cellulose [14,15], lignin is one of the important components of the cell wall of woody plants $[16,17]$. The pulp produces large amounts of by-products containing lignin every year, which provides a comprehensive utilization for lignin in a large number of industries. The special chemical structure of lignin gives its unique function, and it could be added into plasticizers, solubilizers, stabilizers, flame retardants, surfactants, etc. In recent years, preparation of bio-based flame retardants with lignin has received widespread attention due to its high carbon yield after the decomposition of the aromatic framework of lignin. According to reports [18], about $35-40 \%$ of carbon would be produced after pyrolysis of lignin. Taking advantage of this feature, proper addition of lignin to polymers (such as polypropylene, PBS, PET, etc.) could effectively reduce their flammability [19]. In addition, lignin has antioxidant properties and can be added to polymer as a stabilizer to prevent the aging of the polymer. In the past two decades, there have been some reports on lignin-based flame retardants. Some of them are modified by lignin and grafted with some elements containing flame retardant effects such as $\mathrm{P}$ and $\mathrm{N}$, while others are directly compounded with flame retardants [20-23].

Thermogravimetric (TG) analysis is a common method used to analyze biomass pyrolysis behavior and obtain kinetic parameters. Fourier transform infrared spectrometry (FTIR) can be applied to demonstrate the formation and cleavage of chemical bonds, thus indicating the completion of modification. Meanwhile, kinetics is an important factor for determining the mechanism of the thermochemical conversion of biomass. In this paper, two kinetic methods (Flynn-Wall-Ozawa (FWO) and Kissinger-Akahira-Sunose (KAS)) were applied to investigate the kinetics of a lignin-based flame retardant, and the relevant parameters were obtained [24].

\section{Materials and Methods}

\subsection{Materials and Measurement}

Alkaline lignin (Al-lignin) was purchased from TCI Shanghai Co., Ltd., Shanghai, China. Ammonium polyphosphate (APP) and 4,4-diaminodiphenylmethane (DDM) were provided by Shanghai Macklin Co., Ltd., Shanghai, China. Melamine (MEL) was provided by Shanghai Lingfeng Co., Ltd., Shanghai, China. Formaldehyde, $\mathrm{N}, \mathrm{N}$-diemthylformamide (DMF), diethyl ether and ethanol were purchased from Nanjing Chemical Reagent Co., Ltd., Nanjing, China.

TG-FTIR analysis was conducted with a PerkinElmer TGA 8000 thermogravimetric analyzer (PerkinElmer, Hopkinton, MA, USA). The X-ray photoelectron spectroscopy (XPS) spectra were recorded by an AXIS UltraDLD spectrometer with $\mathrm{Al} \mathrm{K \alpha}(1486.6 \mathrm{eV})$ radiation (Shimadzu/Kratos, Kanagawa, Japan). The surface morphologies of the sample were observed by using a JEOLJSM-7600 scanning electron microscopy (SEM) (JEOL, Tokyo, Japan) at the accelerating voltage of $15 \mathrm{kV}$. Limiting oxygen index (LOI) values were measured using a HC-2C oxygen index meter (Jiangsu Institute of Chemical Industry, Jiangsu, China) with a sheet size of $100 \mathrm{~mm} \times 10 \mathrm{~mm} \times 3 \mathrm{~mm}$ according to ASTM D2863-97. Vertical burning (UL-94) was conducted on a CZF-2 instrument (Jiangsu Institute of Chemical Industry, Jiangsu, China) according to ATSM D 3801 standard.

\subsection{Sample Preparation}

Firstly, Al-lignin ( $20 \mathrm{~g}$ ) was firstly added to a flask containing $80 \mathrm{~mL} \mathrm{H}_{2} \mathrm{SO}_{4}(2 \mathrm{~mol} / \mathrm{L})$ solution, heated up to $80^{\circ} \mathrm{C}$ and stirred for $1.5 \mathrm{~h}$. Then, when the temperature was raised to $95^{\circ} \mathrm{C}$, phenol $(18 \mathrm{~g})$ was added, and the mixture was stirred at reflux for $1.5 \mathrm{~h}$. After completion of the reaction, the mixture was washed three times with $500 \mathrm{~mL}$ of diethyl ether and dried at $70{ }^{\circ} \mathrm{C}$ overnight at a constant weight.

Secondly, APP (6 g) was added to a $140 \mathrm{~mL}$ mixture of ethanol to water (5:2) and stirred for 30 $\mathrm{min}$, being then fully dispersed in the solution. Then, MEL $(5 \mathrm{~g})$ was added, the temperature was 
raised to $70{ }^{\circ} \mathrm{C}$ and the mixture was stirred for $6 \mathrm{~h}$. After completion, the mixture was cooled to room temperature, washed repeatedly with ethanol three times, and then dried at $70^{\circ} \mathrm{C}$ for $12 \mathrm{~h}$.

Then $4 \mathrm{~g}$ Ph-lignin and $20 \mathrm{~g}$ MELAPP were added to a flask with $250 \mathrm{~mL}$ DMF as solvent, then $7.2 \mathrm{~g}$ formaldehyde was added at $75^{\circ} \mathrm{C}$ and stirred under reflux for $3 \mathrm{~h}$. After completion of the reaction, $500 \mathrm{~mL}$ of distilled water was added, then the solid was filtered. This was washed three times with $500 \mathrm{~mL}$ distilled water, then dried at $70{ }^{\circ} \mathrm{C}$ under a vacuum for $24 \mathrm{~h}$.

Finally, $3 \mathrm{~g}$ F-lignin@APP and $15 \mathrm{~g}$ EP were mechanically stirred for $1.5 \mathrm{~h}$ at $700 \mathrm{rpm}$ to make the mixture uniform. After stirring, $3 \mathrm{~g}$ DDM was added and stirred for $1.5 \mathrm{~h}$. Then, the bubbles were removed into a vacuum oven and the uniformly mixed resin was poured into the mold, cured at $100^{\circ} \mathrm{C}$ for $2 \mathrm{~h}$, then elevated at $150{ }^{\circ} \mathrm{C}$ for $2 \mathrm{~h}$.

\subsection{Kinetic Modeling}

The kinetic analysis could be obtained through the determination of the kinetic triplets, which are the activation energy (Ea), pre-exponential factor $(\mathrm{A})$ and kinetic model $(\mathrm{f}(\alpha))$. These three kinetic parameters should be determined for complete description of the kinetics for each reaction step. A large number of analytical methods are available nowadays which can be used to determine the kinetic parameters of distinct solid-phase reactions evaluation. The kinetic parameters can be determined either isothermally or nonisothermally by using two main methods: isoconversional (model-free) and model-fitting methods $[25,26]$.

The decomposition process of lignin epoxy resin composite material is a solid state reaction process $[27,28]$. Generally, the rate of thermal decomposition can be expressed as a function of the degree of conversion $f(\alpha)$ and temperature $K(T)$, as shown in Equation (1) [29]:

$$
\frac{d \alpha}{d t}=\beta \frac{d \alpha}{d T}=K(T) f(\alpha) h(P)
$$

where $\alpha$ is the conversion rate (\%) of the sample in the reaction temperature range, $\beta$ is the linear heating rate $\left({ }^{\circ} \mathrm{C} / \mathrm{min}\right), T$ is the reaction temperature $(K)$ and $P$ is the pressure $(\mathrm{Pa})$. Although pressure may have an impact on kinetic analysis, pressure is usually ignored in pyrolysis kinetic analysis of solid state reactions [29,30]. After ignoring the pressure, Equation (1) can be written as a function containing two variables $(\alpha$ and $T)$ :

$$
\frac{d \alpha}{d t}=\beta \frac{d \alpha}{d T}=K(T) f(\alpha)
$$

The conversion rate $(\alpha)$ can be obtained by Equation (3):

$$
\alpha=\left(M_{0}-M_{T}\right) /\left(M_{0}-M_{\infty}\right)
$$

where $M_{0}$ is the initial mass of the sample, $M_{T}$ is the mass of the sample residue at the corresponding temperature and $M_{\infty}$ is the mass of the sample residue at the final temperature. The temperature-dependent equation $K(T)$ is derived from the Arrhenius equation:

$$
K(T)=A \exp (-E / R T)
$$

where $A$ and $E$ are kinetic parameters. $A$ is the pre-exponential factor, $E$ is apparent activation energy $(\mathrm{kJ} / \mathrm{mol})$ and $R$ is the gas constant $(8.314 \mathrm{~J} /(\mathrm{mol} \cdot \mathrm{K}))$.

Bring Equation (4) into Equation (1), separate the variables and integrate the equation to get Equation (5):

$$
G(\alpha)=\int_{0}^{\alpha} d \alpha / f(x)=\frac{A}{B} \int_{0}^{T} \exp (-E / R T) d T=(A E / \beta R) P(u)
$$


where $G(\alpha)$ is the integral form of $f(x), u$ is usually defined as the temperature integral of $E / R T$ and $P(u) . P(u)$ has many mathematical assumptions to determine the activation energy.

In the absence of a kinetic model, there are several ways to obtain the activation energy during the reaction. Usually these methods are divided into the differential method and integral method. The integral method usually deviates greatly from the actual value. Compared with the integral method, the differential method does not involve approximate values, so the results obtained are more accurate.

Kissinger-Akahira-Sunoe (KAS) and Flynn-Wall-Ozawa (FWO) are two methods commonly used to obtain the activation energy of thermal decomposition:

$$
\begin{gathered}
\ln \left(\beta / T^{2}\right)=\ln (A R / E G(\alpha))-E / R T \\
\ln (\beta)=\ln (A R / E G(\alpha))-2.315-0.4567 E / R T
\end{gathered}
$$

According to the KAS and FWO methods, the activation energy for a specific value can be obtained from the slopes of plot $\ln \left(\beta / T^{2}\right)$ vs. $1 / T$ and plot $\log (\beta)$ vs. $1 / T$, respectively, for a set of 4 heating rates $(\beta)$. It should be noted that $T$ is strongly dependent on $\beta$ at a specific value, as discussed previously.

\subsection{Characterization}

Fourier transform infrared (FTIR) spectra were recorded by a Nicolet 6700 FTIR spectrometer under the resolution of $1 \mathrm{~cm}^{-1}$ in 32 scans by a $\mathrm{KBr}$ disk with the wavenumber ranging from 4000 to $500 \mathrm{~cm}^{-1}$. Thermogravimetric analysis (TG) was obtained on a DTG-60AH (SHIMADZU, Japan). The epoxy resin composites (about $5 \mathrm{mg}$ ) were heated from 30 to $700{ }^{\circ} \mathrm{C}$ at a rate of $15^{\circ} \mathrm{C} \mathrm{min}^{-1}$ under the nitrogen flow of $40 \mathrm{~mL} \cdot \mathrm{min}^{-1}$. The TG-FTIR analysis was conducted on a thermogravimetric analysis (TGA, Perkin Elmer Pyris 1 TGA unit), coupled with a Fourier transform infrared spectrometer. The X-ray photoelectron spectroscopy (XPS) spectra were recorded by an AXIS UltraDLD spectrometer with $\mathrm{Al} \mathrm{K} \alpha(1486.6 \mathrm{eV})$ radiation.

\section{Results and Discussion}

\subsection{Characterization of F-Lignin@APP}

The FTIR spectra of Al-lignin, Ph-lignin and F-lignin@APP are shown in Figure 1. From Figure 1, we can observe that the basic structure of lignin does not change much after phenolation. Aromatic skeletal stretching vibrations shift from 1600 and $1503 \mathrm{~cm}^{-1}$ to 1624 and $1512 \mathrm{~cm}^{-1}, \mathrm{C}-\mathrm{H}$ bending and out-of-plane deform vibrations shift from 1460 and $858 \mathrm{~cm}^{-1}$ to 1458 and $873 \mathrm{~cm}^{-1}$ [31]. The peak at $1222 \mathrm{~cm}^{-1}$ is assigned to the syringyl structure. Its absorption peak intensity is enhanced, indicating that the phenolization of lignin was successful [32]. After F-lignin@APP wrapped APP, some absorption peaks appeared, such as the absorption peak of the $\mathrm{N}-\mathrm{H}$ bending vibration in the $\mathrm{NH}_{2}$ structure at 1640 $\mathrm{cm}^{-1}$, the absorption peak of the triazine ring at $813 \mathrm{~cm}^{-1}$ and the absorption peak of the stretching vibration $\mathrm{P}-\mathrm{O}$ at $1088 \mathrm{~cm}^{-1}$ [33]. 


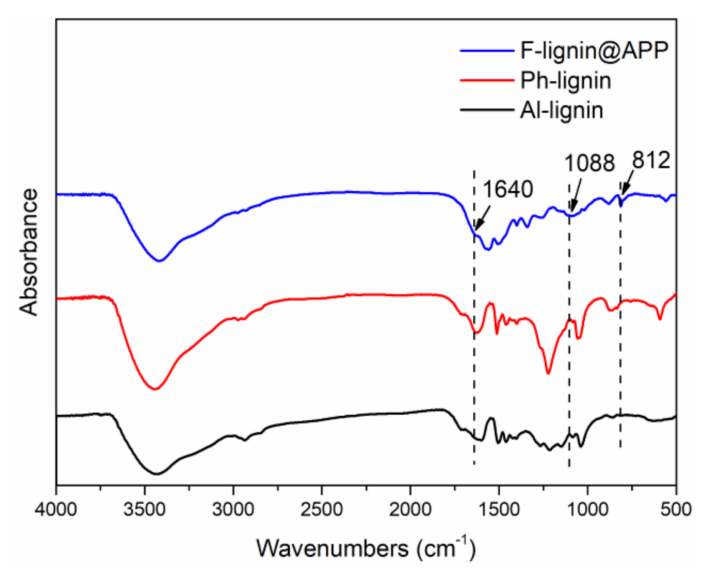

Figure 1. FTIR spectra of Al-lignin, Ph-lignin and F-lignin@APP.

The chemical composition and types of chemical bonds can be analyzed by XPS spectra. The $\mathrm{N}_{1 \mathrm{~s}}$ spectra of the MEL and F-lignin@APP are shown in Figure 2. It can be seen from Figure 2a that the peak at $401.6 \mathrm{eV}$ represents $\mathrm{NH}_{2}$ in the MEL and the peak at $399.3 \mathrm{eV}$ may stand for the nitrogen in the $\mathrm{C}=\mathrm{N}$ double bonds of the MEL [34]. As can be observed from Figure 2b, the peak of $\mathrm{C}=\mathrm{N}$ double bond in the MEL still appears at $399.3 \mathrm{eV}$. After functionalization, two new peaks appeared; the peak at 401.2 $\mathrm{eV}$ corresponds to $\mathrm{NH}_{4}{ }^{+}$in the APP. Another peak at $398.1 \mathrm{eV}$ should be attributed to the $\mathrm{N}$ in the -NH- for F-lignin@APP [35].
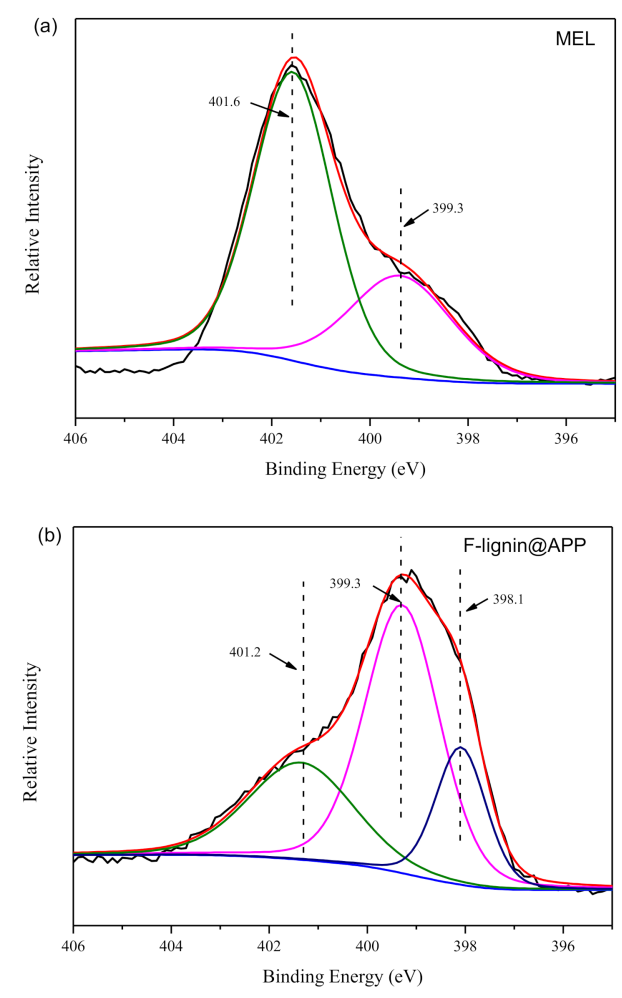

Figure 2. $\mathrm{N}_{1 \mathrm{~s}}$ spectra of (a) melamine (MEL) and (b) F-lignin@APP.

The thermogravimetry (TG) and derivative thermogravimetry (DTG) curves of Al-lignin, Ph-lignin and F-lignin@APP are shown in Figure 3. As can be observed from Figure 3a, the carbon residues of the Ph-lignin and F-lignin@APP were reduced compared with the Al-lignin: i.e., 33.5\% and 37.5\%, respectively. In addition, the DTG curves of the three samples all showed a peak before $150{ }^{\circ} \mathrm{C}$ due to evaporation of the residual moisture contained in the three samples. Different from the Al-lignin and 
F-lignin@APP, a broad endothermic peak was observed for the Ph-lignin at $160-320^{\circ} \mathrm{C}$, which might be formed by the decomposition and volatilization of lignin with lower molecular weight. At $320-500{ }^{\circ} \mathrm{C}$, an endothermic peak appeared for the three samples, because the bonds between the structural units of lignin were broken and some monomer phenols evaporated. In addition, it could be found that the endothermic peak of the F-lignin@APP is much stronger than the other samples due to the degradation of the lignin and the decomposition of the APP during the heating process.
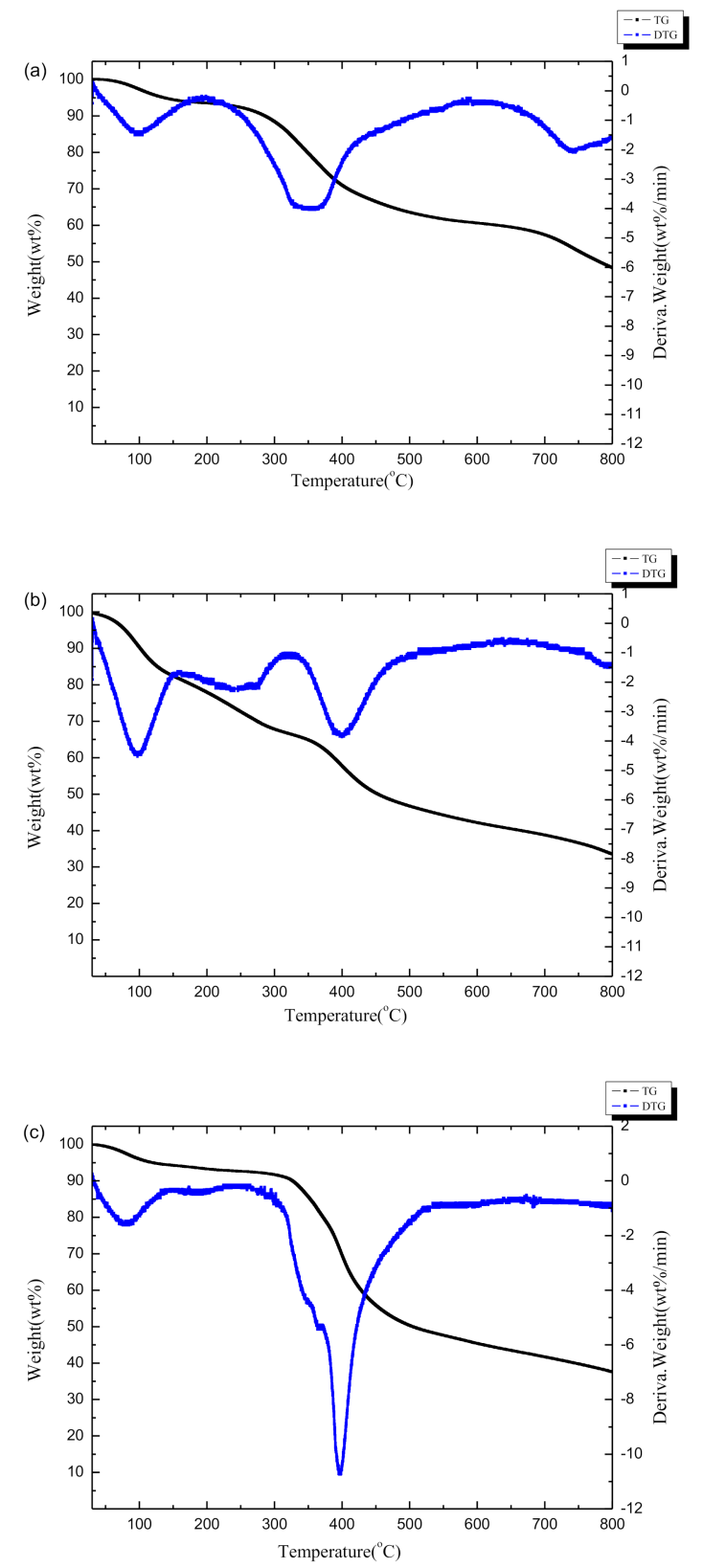

Figure 3. Thermogravimetry (TG) and derivative thermogravimetry (DTG) curves of (a) Al-lignin, (b) Ph-lignin and (c) F-lignin@APP.

The FTIR spectra of volatile products originating from Al-lignin, Ph-lignin and F-lignin@APP are shown in Figure 4. The characteristic peaks of F-lignin@APP are indicated at 3330, 2356, 1593, $1509,1100,962$ and $928 \mathrm{~cm}^{-1}$. Among them, the peaks at $3330 \mathrm{~cm}^{-1}$ and $1593 \mathrm{~cm}^{-1}$ are absorption peaks of $\mathrm{NH}_{3}$. The peaks at 1100, 962 and $928 \mathrm{~cm}^{-1}$ illustrate the presence of phosphoric acid derivatives, which are attributed to $\mathrm{P}-\mathrm{O}-\mathrm{C}, \mathrm{P}-\mathrm{O}-\mathrm{Ph}$ and $\mathrm{P}-\mathrm{O}-\mathrm{P}$, respectively $[20,36]$. Compared 
with Ph-lignin and Al-lignin, the absorption peaks of F-lignin@APP do not contain hydrocarbon compounds, carbonyl compounds and aromatic compounds, which are usually formed by breaking the chemical bonds of functional groups on the basic structural units of lignin. It was concluded that the depolymerization products of lignin synergized with the polyacid generated by APP decomposition would form phosphorus-containing derivatives.

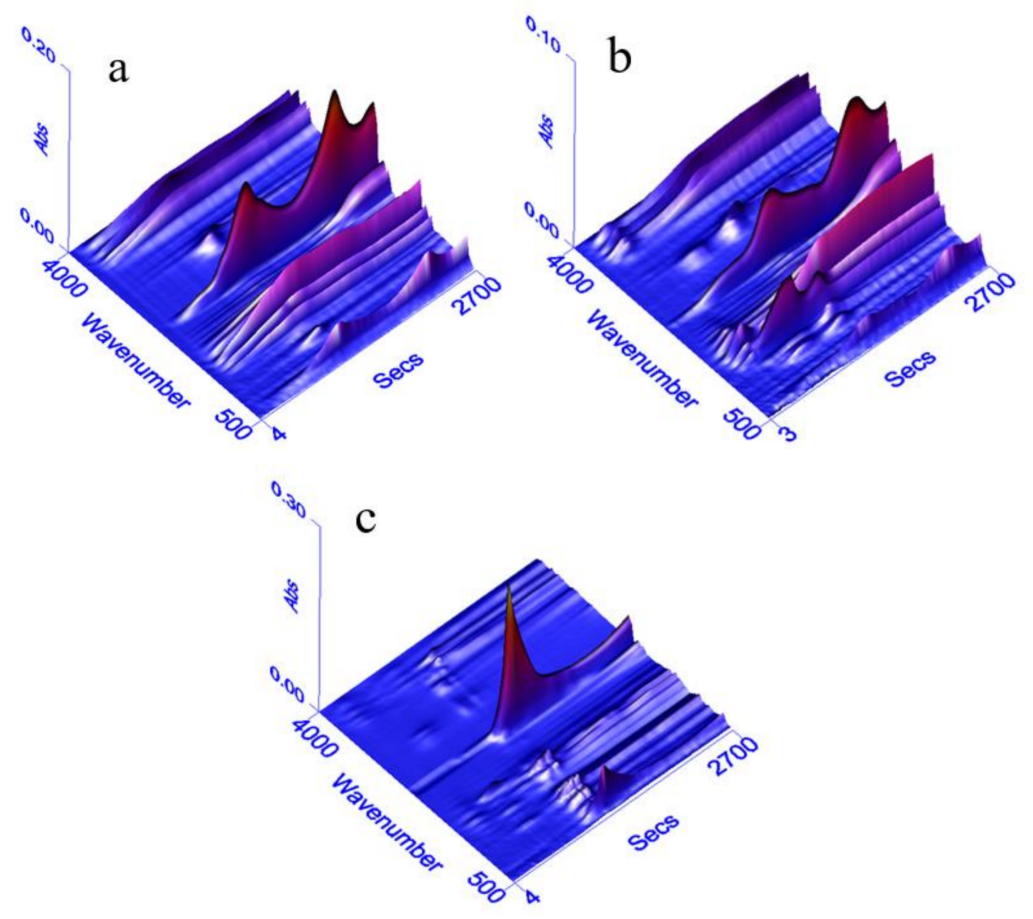

Figure 4. 3D FTIR spectra of the volatile products of Al-lignin (a), Ph-lignin (b) and F-lignin@APP (c).

\subsection{Thermal and Fire Properties of F-Lignin@APP 20/EP}

The degradation process of EP, Al-lignin 20/EP and F-lignin@APP 20/EP under an $\mathrm{N}_{2}$ atmosphere is shown in Figure 5 and the detailed data are listed in Table 1. From Figure 5 , the $\mathrm{T}_{\mathrm{dmax}}$ (the temperature of the maximum decomposition rate) of EP, Al-lignin 20/EP and F-lignin@APP 20/EP are 405, 401 and $382{ }^{\circ} \mathrm{C}$, respectively. Due to the presence of APP in the flame retardant, the degradation temperature of APP is lower than that of F-lignin@APP. The maximum decomposition rate of F-lignin@APP 20/EP is $11.8 \% / \mathrm{min}$, which is $8 \% / \mathrm{min}$ and $4.7 \% / \mathrm{min}$ lower than EP and Al-lignin. In addition, the char residue of F-lignin@APP 20/EP is 32.5\%, which is much higher than EP. Lower decomposition rate and higher char residue might effectively improve the thermal stability of EP modified by F-lignin@APP. 

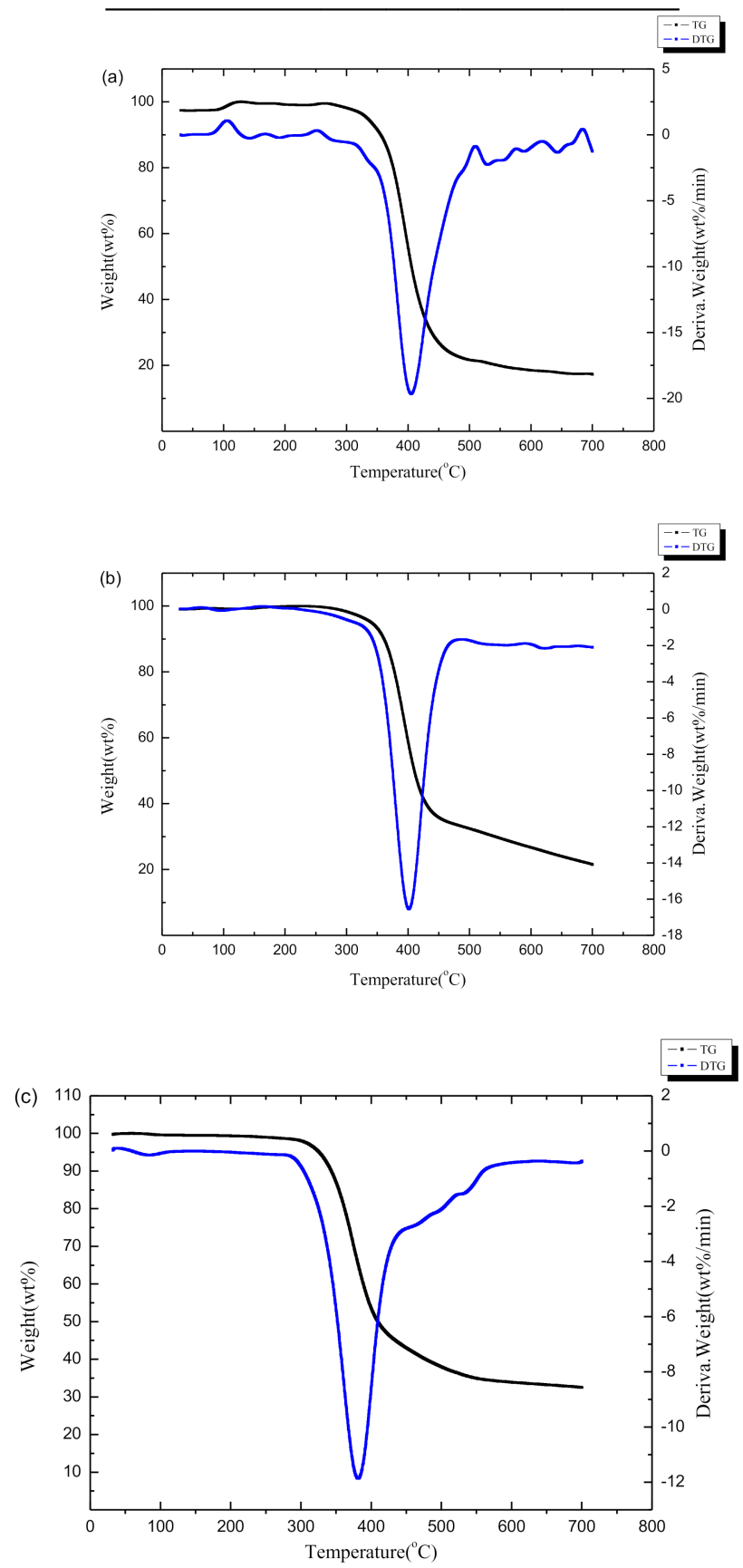

Figure 5. TG and DTG curves of (a) EP, (b) Al-lignin20/EP and (c) F-lignin@APP20/EP.

Table 1. Thermogravimetric analysis (TGA) data of epoxy resin (EP), Al-lignin 20/EP and F-lignin@APP20/EP in $\mathrm{N}_{2}$.

\begin{tabular}{ccccc}
\hline \multirow{2}{*}{ Samples } & $\mathbf{T}_{\mathbf{d 5} \%}$ & $\mathbf{T}_{\mathbf{d m a x}}$ & $\mathbf{d W / d T}$ & \multicolumn{1}{c}{ Residues } \\
\cline { 2 - 5 } & $\mathbf{(} \mathbf{C})$ & $\mathbf{(}{ }^{\circ} \mathbf{C}$ & $\mathbf{( \% / m i n )}$ & $\mathbf{( \% )}$ \\
\hline EP & 333 & 405 & 19.8 & 17.2 \\
Al-lignin 20/EP & 340 & 401 & 16.5 & 21.5 \\
F-lignin@APP20/EP & 325 & 382 & 11.8 & 32.5 \\
\hline
\end{tabular}

In order to investigate the flame retardance of the EPs, the LOI tests and vertical burning tests (UL-94) were carried out at the room temperature, and the results are shown in Table 2. As shown in Table 2, the LOI of EP is only 26.7\%. As the content of F-lignin@APP increases, the LOI of the EP reaches 
$34.7 \%$ and a V1 classification (good flame retardancy) at the thickness of $3 \mathrm{~mm}$. While the content of F-lignin@APP reaches $20 \mathrm{wt} \%$, the result of the LOI test indicates that the F-lignin@APP20/EP gets an LOI (36.1\%) that is much higher than the LOI of EP. Moreover, it is easy to reach a V0 classification (excellent flame retardancy) with $20 \mathrm{wt} \%$ of the F-lignin@APP added.

Table 2. Flame retardance of EP, F-lignin@APP18/EP and F-lignin@APP20/EP.

\begin{tabular}{cccc}
\hline Sample & EP & F-Lignin@APP18/EP & F-Lignin@APP20/EP \\
\hline LOI $(\%)$ & 26.7 & 34.7 & 36.1 \\
UL-94 rating & - & V1 & V0 \\
\hline
\end{tabular}

\subsection{Characterization of Gas Phase}

FTIR spectra of the volatilities of EP, Al-lignin 20/EP and F-lignin@APP 20/EP at $\mathrm{T}_{\mathrm{dmax}}$ are shown in Figure 6. We can observe that they have several of the same absorption peaks: e.g., the peak near $3650 \mathrm{~cm}^{-1}$ is the absorption peak of $\mathrm{NH}_{3}$ and $\mathrm{H}_{2} \mathrm{O}$ alike. The peak at $2975 \mathrm{~cm}^{-1}$ is attributed to the stretching vibration absorption peak of the hydrocarbon [31]. The peak at $2358 \mathrm{~cm}^{-1}$ is assigned to the stretching vibration absorption peak of the $\mathrm{C}=\mathrm{O}$ in the $\mathrm{CO}_{2}$. The peak at $1750 \mathrm{~cm}^{-1}$ is the absorption peak of the carbonyl component, and the peaks near $1515 \mathrm{~cm}^{-1}$ are the absorption peaks of the aromatic component [37]. The peaks around $1170 \mathrm{~cm}^{-1}$ are mainly the absorption peaks of the $\mathrm{C}-\mathrm{C}$ and $\mathrm{C}-\mathrm{O}$ originating from the structure of the phenols and ethers. These absorption peaks are closely related to the epoxy resin, i.e., bisphenol A type, and the use of the amino group containing a generated curing agent (DDM) [38]. Unlike Al-lignin 20/EP and EP, F-lignin@APP 20/EP has several new absorption peaks. The peak at $3330 \mathrm{~cm}^{-1}$ is the absorption peak of $\mathrm{NH}_{2}$ in the melamine. The peaks at 962 and 928 $\mathrm{cm}^{-1}$ are the absorption peak of the phosphorus-containing derivatives, $\mathrm{P}-\mathrm{O}-\mathrm{P}$ and $\mathrm{P}-\mathrm{O}$, respectively. In addition, the absorption peak intensity of the carbonyl compound decreases. It is indicated that F-lignin@APP 20/EP can produce polyacids and $\mathrm{NH}_{3}$ during pyrolysis. These polyacids interact with the carbon produced by the decomposition of lignin to form a condensed phase, which could block some of the volatiles to some extent.

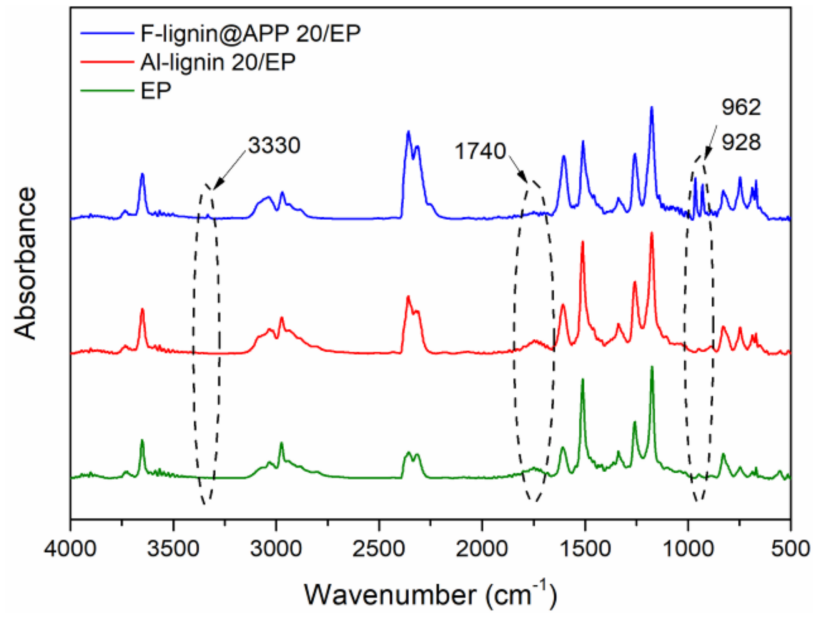

Figure 6. FTIR spectra of the volatile products of EP, Al-lignin 20/EP and F-lignin@APP20/EP.

\subsection{Kinetics Analysis}

Al-lignin 20/EP (Figure 7) and F-lignin@APP20/EP (Figure 8) pyrolysis kinetic parameters were estimated by KAS method and FWO method. The activation energy at different conversion rates obtained from both methods is shown in Table 3and Table 4. The coefficient $\left(R^{2}\right)$ of the composite materials methods was around 0.99 , which meant the data fit well. In addition, the difference in 
activation energy data obtained by KAS and FWO methods was very small and this means that the estimated value was relatively reliable.

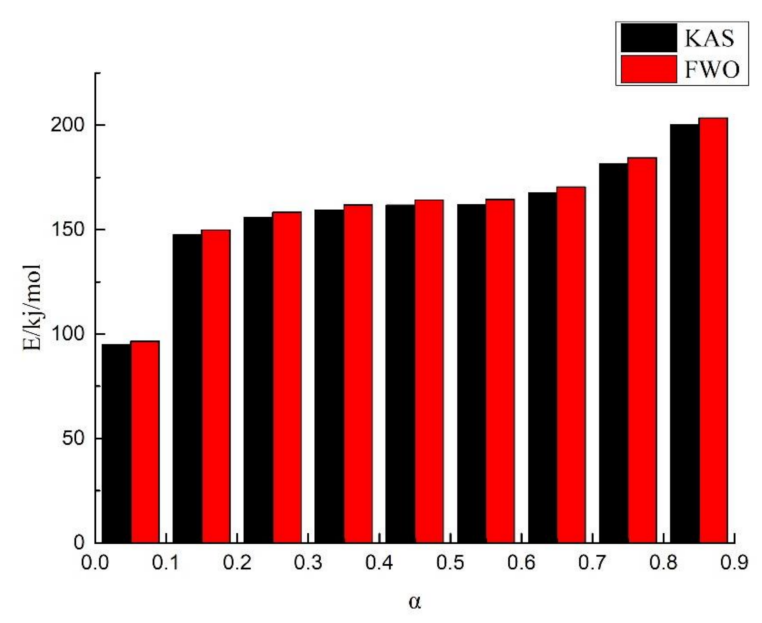

Figure 7. Histogram of Kissinger-Akahira-Sunose (KAS) method and Flynn-Wall-Ozawa (FWO) method for Al-lignin 20/EP.

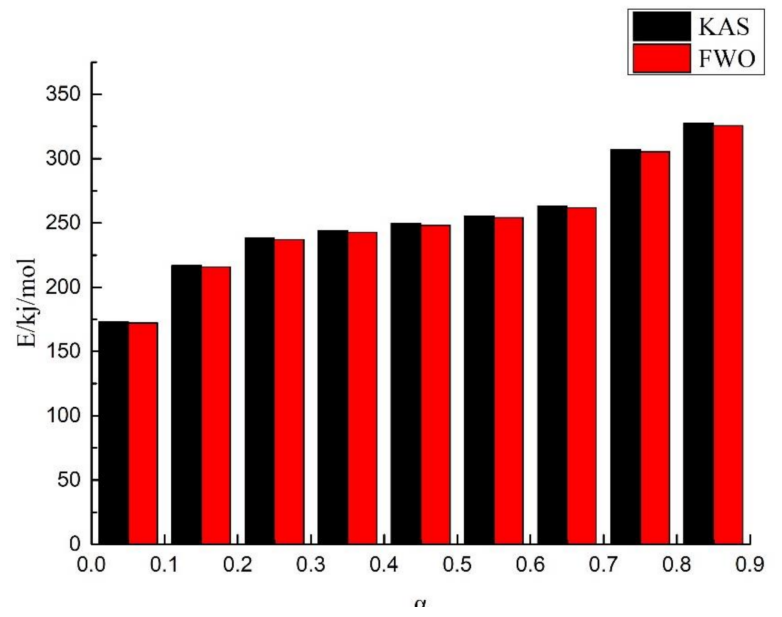

Figure 8. Histogram of KAS method and FWO method for F-lignin@APP20/EP.

Table 3. Activation energy of Al-lignin 20/EP obtained from the KAS method and FWO method.

\begin{tabular}{ccc}
\hline $\boldsymbol{\alpha}$ & KAS $\left(\mathbf{R}^{\mathbf{2}}\right)$ & FWO $\left(\mathbf{R}^{\mathbf{2}}\right)$ \\
\hline 0.05 & $95.00(0.98)$ & $96.52(0.99)$ \\
0.15 & $147.49(0.98)$ & $149.87(0.99)$ \\
0.25 & $155.76(0.99)$ & $158.26(0.99)$ \\
0.35 & $159.29(0.99)$ & $161.84(0.99)$ \\
0.45 & $161.66(0.99)$ & $164.25(0.99)$ \\
0.55 & $161.94(0.99)$ & $164.54(0.99)$ \\
0.65 & $167.66(0.99)$ & $170.36(0.99)$ \\
0.75 & $181.48(0.99)$ & $184.40(0.99)$ \\
0.85 & $200.22(0.98)$ & $203.42(0.99)$ \\
\hline
\end{tabular}


Table 4. Activation energy of F-lignin@APP20/EP obtained from the KAS method and FWO method.

\begin{tabular}{ccc}
\hline $\boldsymbol{\alpha}$ & KAS $\left(\mathbf{R}^{\mathbf{2}} \mathbf{)}\right.$ & FWO $\left(\mathbf{R}^{\mathbf{2}}\right)$ \\
\hline 0.05 & $173.28(0.99)$ & $172.39(0.99)$ \\
0.15 & $217.00(0.97)$ & $215.80(0.98)$ \\
0.25 & $238.31(0.99)$ & $237.11(0.99)$ \\
0.35 & $243.94(0.99)$ & $242.71(0.99)$ \\
0.45 & $249.63(0.99)$ & $248.37(0.99)$ \\
0.55 & $255.39(0.99)$ & $254.11(0.99)$ \\
0.65 & $263.17(0.99)$ & $261.85(0.99)$ \\
0.75 & $307.06(0.98)$ & $305.36(0.98)$ \\
0.85 & $327.45(0.99)$ & $325.80(0.99)$ \\
\hline
\end{tabular}

The activation energy of Al-lignin 20/EP and F-lignin@APP 20/EP at different conversion rates is shown in Figure 9. It can be seen that the thermal decomposition of both materials is a very complicated process, accompanied by many reactions. Combined with the gas phase analysis, the pyrolysis process of Al-lignin 20/EP and F-lignin@APP 20/EP can be divided into three stages. In stage I, the activation energy required for pyrolysis is low. During this stage, the activation energy required for the cleavage of some functional groups in the lignin structure, e.g., hydroxyl, methyl and methoxy groups, is low [39]. In addition, the APP might decompose, producing some phosphorus-containing derivatives and $\mathrm{NH}_{3}$ at this stage. In stage II, the lignin will depolymerize at a higher temperature. The thermal stability of the ether bond is lower than that of the $\mathrm{C}-\mathrm{C}$ bond, so this kind of bond will be broken first [40], producing more ether compounds in volatiles. In addition, the decomposition products of the APP will also interact with the depolymerization products of the lignin at this stage. Therefore, the growth rate of the activation energy of F-lignin@APP 20/EP is higher than that of Al-lignin 20/EP, as shown in Figure 9. In stage III, the growth rate of the activation energy of F-lignin@APP 20/EP is much higher than that of Al-lignin 20/EP, indicating that the carbon layer formed by F-lignin@APP 20/EP is more stable than Al-lignin 20/EP.

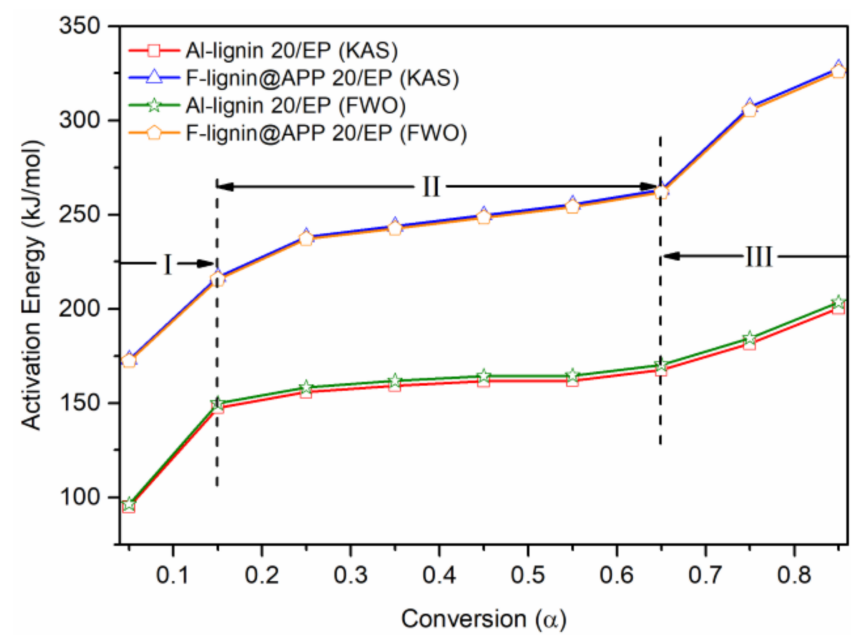

Figure 9. Activation energy of Al-lignin 20/EP and F-lignin@APP20/EP at different conversion.

\section{Conclusions}

A novel lignin-based flame retardant (F-lignin@APP) containing P and N was successfully prepared. TG-DTG results showed that the maximum decomposition rate of F-lignin@APP 20/EP was $11.8 \% / \mathrm{min}$, which was $8 \% / \mathrm{min}$ and $4.7 \% / \mathrm{min}$ lower than that of EP and Al-lignin. In addition, the char residue of F-lignin@APP 20/EP was 32.5\%, much higher than that of EP. Moreover, the pyrolysis process of Al-lignin 20/EP and F-lignin@APP 20/EP can be divided into three stages. F-lignin@APP 
played an important part during stage III, leading to a good flame retardant performance due to the carbon layer formed at a high temperature.

Author Contributions: X.L. and Q.H. contributed equally. X.L. and Q.H. carried out the literature search, data collection and data analysis. X.L., Q.H. and X.W. carried out data collection and data analysis. L.L. and Y.D. carried out data collection and figures drawing. C.S. and C.H. did data interpretation and wrote the manuscript. X.G. designed the framework and wrote the manuscript. All authors discussed the results and contributed to the final manuscript. All authors have read and agreed to the published version of the manuscript.

Funding: This research was funded by the National Natural Science Foundation of China (no. 21774059), the Priority Academic Program Development (PAPD) of Jiangsu Higher Education Institutions, the opening funding of Jiangsu Key Lab of Biomass-based Green Fuels and Chemicals and the College Students' Practice and Innovation Training Project (202010298007Z).

Conflicts of Interest: The authors declare no competing financial interest.

\section{References}

1. Tan, Y.; Shao, Z.B.; Chen, X.F.; Long, J.W.; Chen, L.; Wang, Y.Z. Novel multifunctional organic-inorganic hybrid curing agent with high flame-retardant efficiency for epoxy resin. ACS Appl. Mater. Interfaces 2015, 7, 17919-17928. [CrossRef] [PubMed]

2. Sun, J.; Wang, X.D.; Wu, D.Z. Novel spirocyclic phosphazene-based epoxy resin for halogen-free fire resistance: Synthesis, curing behaviors, and flammability characteristics. ACS Appl. Mater. Interfaces 2012, 4, 4047-4061. [CrossRef] [PubMed]

3. Xu, Y.J.; Chen, L.; Rao, W.H.; Qi, M.; Guo, D.M.; Liao, W.; Wang, Y.Z. Latent curing epoxy system with excellent thermal stability, flame retardance and dielectric property. Chem. Eng. J. 2018, 347, 223-232. [CrossRef]

4. Xu, Y.J.; Wang, J.; Tan, Y.; Qi, M.; Chen, L.; Wang, Y.Z. A novel and feasible approach for one-pack flame-retardant epoxy resin with long pot life and fast curing. Chem. Eng. J. 2018, 337, 30-39. [CrossRef]

5. Liu, J.K.; Dai, J.Y.; Wang, S.P.; Peng, Y.Y.; Cao, L.J.; Liu, X.Q. Facile synthesis of bio-based reactive flame retardant from vanillin and guaiacol for epoxy resin. Compos. Part B 2020, 190, 107926. [CrossRef]

6. Baruah, P.; Karak, N. Bio-based tough hyperbranched epoxy/graphene oxide nanocomposite with enhanced biodegradability attribute. Polym. Degrad. Stab. 2016, 129, 26-33. [CrossRef]

7. Levchik, S.V.; Weil, E.D. Thermal decomposition, combustion and flame-retardancy of epoxy resins? A review of the recent literature. Polym. Int. 2004, 53, 1901-1929. [CrossRef]

8. Barral, L.; Cano, J.; López, J.; López-Bueno, I.; Nogueira, P.; Abad, M.J.; Ramírez, C. Decomposition behavior of epoxy-resin systems cured by diamines. Eur. Polym. J. 2000, 36, 1231-1240. [CrossRef]

9. Dyakonov, T.; Mann, P.J.; Chen, Y.; Stevenson, W.T.K. Thermal analysis of some aromatic amine cured model epoxy resin systems-II: Residues of degradation. Polym. Degrad. Stab. 1996, 54, 67-83. [CrossRef]

10. Mustafa, M.F; Cook, W.D.; Schiller, T.L.; Siddiqi, H.M. Curing behavior and thermal properties of TGDDM copolymerized with a new pyridine-containing diamine and with DDM or DDS. Thermochim. Acta 2014, 575, 21-28. [CrossRef]

11. Xiao, W.; He, P.; Hu, G.; He, B. Study on the flame-retardance and thermal stability of the acid anhydride-cured epoxy resin flame-retarded by triphenyl phosphate and hydrated alumina. J. Fire Sci. 2001, 19, 369-377.

12. Kodolov, V.I.; Shuklin, S.G.; Kuznetsov, A.P.; Makarova, L.G.; Bystrov, S.G.; Demicheva, O.V.; Rudakova, T.A. Formation and investigation of epoxy intumescent compositions modified by active additives. J. Appl. Polym. Sci. 2002, 85, 1477-1483.

13. Liu, W.; Varley, R.J.; Simon, G.P. A phosphorus-containing diamine for flame-retardant, high-functionality epoxy resins I. synthesis, reactivity, and thermal degradation properties. J. Appl. Polym. Sci. 2004, 92, 2093-2100.

14. Cao, L.C.; Yu, I.K.M.; Liu, Y.Y.; Ruan, X.X.; Daniel, C.W.T.; Hunt, A.J.; Ok, Y.S.; Song, H.; Zhang, S.C. Lignin valorization for the production of renewable chemicals: State-of-the-art review and future prospects. Bioresour. Technol. 2018, 269, 465-475.

15. Gonçalves, A.R.; Benar, P. Hydroxymethylation and oxidation of organosolv lignins and utilization of the products. Bioresour. Technol. 2001, 79, 103-111.

16. Hu, J.; Xiao, R.; Shen, D.K.; Zhang, H.Y. Structural analysis of lignin residue from black liquor and its thermal performance in thermogravimetric-Fourier transform infrared spectroscopy. Bioresour. Technol. 2013, 128, 633-639. 
17. Kumar, A.; Kumar, A.; Kumar, J.; Bhaskar, T. Catalytic pyrolysis of soda lignin over zeolites using pyrolysis gas chromatography-mass spectrometry. 2019, 291, 121822.

18. Li, B.; Zhang, X.C.; Su, R.Z. An investigation of thermal degradation and charring of larch lignin in the condensed phase: The effects of boric acid, guanyl urea phosphate, ammonium dihydrogen phosphate and ammonium polyphosphate. Polym. Adv. Technol. 2002, 1, 35-44.

19. Gregorova, A.; Kosikova, R.; Moravcik, R. Stabilization effect of lignin in natural rubber. Polym. Degrad. Stab. 2006, 91, 229-233.

20. Liu, L.N.; Huang, G.B.; Song, P.A.; Yu, Y.M.; Fu, S.Y. Converting industrial alkali lignin to biobased functional additives for improving fire behavior and smoke suppression of polybutylene succinate. ACS Sustain. Chem. Eng. 2016, 4, 4732-4742.

21. Liu, L.N.; Qian, M.B.; Song, P.A.; Huang, G.B.; Yu, Y.M.; Fu, S.Y. Fabrication of green lignin-based flame retardants for enhancing the thermal and fire retardancy properties of polypropylene/wood composites. ACS Sustain. Chem. Eng. 2016, 4, 2422-2431. [CrossRef]

22. Wu, W.; He, H.B.; Liu, T.; Wei, R.C.; Cao, X.W.; Sun, Q.J.; Venkatesh, S.; Yuen, R.K.K.; Roy, V.A.L.; Li, R.K.Y. Synergetic enhancement on flame retardancy by melamine phosphate modified lignin in rice husk ash filled P34HB biocomposites. Compos. Sci. Technol. 2018, 168, 246-254. [CrossRef]

23. Costes, L.; Laoutid, F.; Aguedo, M.; Richel, A.; Brohez, S.; Delvosalle, C.; Dubois, P. Phosphorus and nitrogen derivatization as efficient route for improvement of lignin flame retardant action in PLA. Eur. Polym. J. 2016, 84, 652-667. [CrossRef]

24. Lu, X.Y.; Dai, P.; Zhu, X.J.; Guo, H.Q.; Que, H.; Wang, D.D.; Liang, D.X.; He, T.; Dong, Y.G.; Li, L.; et al. Thermal behavior and kinetics of enzymatic hydrolysis lignin modified products. Thermochim. Acta 2020, 688,178593 . [CrossRef]

25. Khawam, A.; Flanagan, D.R. Basics and applications of solid-state kinetics: A pharmaceutical perspective. J. Pharm. Sci. 2006, 95, 472-498. [CrossRef]

26. Zhang, L.K.; Zheng, X.Y. Experimental study on thermal decomposition kinetics of natural ageing AP/HTPB base bleed composite propellant. Defence Technol. 2018, 14, 422-425. [CrossRef]

27. Ferdosian, F.; Yuan, Z.S.; Anderson, M.; Xu, C.C. Thermal performance and thermal decomposition kinetics of lignin-based epoxy resins. J. Anal. Appl. Pyrolysis 2016, 119, 124-132. [CrossRef]

28. Vasudev, V.; Ku, X.L.; Lin, J.Z. Kinetic study and pyrolysis characteristics of algal and lignocellulosic biomasses. Bioresour. Technol. 2019, 288, 121496. [CrossRef]

29. Vyazovkin, S.; Burnham, A.K.; Criado, J.M.; Pérez-Maqueda, L.A.; Popescu, C.; Sbirrazzuoli, N. ICTAC Kinetics Committee recommendations for performing kinetic computations on thermal analysis data. Thermochim. Acta 2011, 520, 1-19. [CrossRef]

30. Qin, K.; Thunman, H. Diversity of chemical composition and combustion reactivity of various biomass fuels. Fuel 2015, 147, 161-169. [CrossRef]

31. Wang, S.R.; Ru, B.; Lin, H.Z.; Sun, W.X.; Luo, Z.Y. Pyrolysis behaviors of four lignin polymers isolated from the same pine wood. Bioresour. Technol. 2015, 182, 120-127. [CrossRef] [PubMed]

32. Jiao, G.J.; Peng, P.; Sun, S.L.; Geng, Z.C.; She, D. Amination of biorefinery technical lignin by Mannich reaction for preparing highly efficient nitrogen fertilizer. Int. J. Biol. Macromol. 2019, 127, 544-554. [CrossRef] [PubMed]

33. Jin, X.D.; Sun, J.; Zhang, J.S.Q.; Gu, X.Y.; Bourbigot, S.; Li, H.F.; Tang, W.F.; Zhang, S. Preparation of a novel intumescent flame retardant based on supramolecular interactions and its application in polyamide 11. ACS Appl. Mater. Interfaces 2017, 9, 24964-24975. [CrossRef] [PubMed]

34. Guan, Y.H.; Huang, J.Q.; Yang, J.C.; Shao, Z.B.; Wang, Y.Z. An effective way to flame-retard biocomposite with ethanolamine modified ammonium polyphosphate and its flame retardant mechanisms. Ind. Eng. Chem. Res. 2015, 54, 3524-3531. [CrossRef]

35. Shao, Z.B.; Deng, C.; Tan, Y.; Chen, M.J.; Chen, L.; Wang, Y.Z. An efficient mono-component polymeric intumescent flame retardant for polypropylene: Preparation and application. ACS Appl. Mater. Interfaces 2014, 6, 7363-7370. [CrossRef]

36. Xiong, Y.Q.; Jiang, Z.J.; Xie, Y.Y.; Zhang, X.Y.; Xu, W.J. Development of a DOPO-containing melamine epoxy hardeners and its thermal and flame-retardant properties of cured products. J. Appl. Polym. Sci. 2013, 127, 4352-4358. [CrossRef] 
37. Ma, Z.Q.; Wang, J.H.; Zhou, H.Z.; Zhang, Y.; Yang, Y.Y.; Liu, X.H.; Ye, J.W.; Chen, D.Y.; Wang, S.R. Relationship of thermal degradation behavior and chemical structure of lignin isolated from palm kernel shell under different process severities. Fuel Process. Technol. 2018, 181, 142-156. [CrossRef]

38. Lascano, D.; Quiles-Carrillo, L.; Balart, R.; Boronat, T.; Montanes, N. Kinetic analysis of the curing of a partially biobased epoxy resin using dynamic differential scanning calorimetry. Polymers 2019, 11, 391. [CrossRef]

39. Ma, Z.Q.; Sun, Q.F.; Ye, J.W.; Yao, Q.F.; Zhao, C. Study on the thermal degradation behaviors and kinetics of alkali lignin for production of phenolic-rich bio-oil using TGA-FTIR and Py-GC/MS. J. Anal. Appl. Pyrolysis 2016, 117, 116-124. [CrossRef]

40. Shen, D.K.; Liu, G.F.; Zhao, J.; Xue, J.T.; Guan, S.P.; Xiao, R. Thermo-chemical conversion of lignin to aromatic compounds: Effect of lignin source and reaction temperature. J. Anal. Appl. Pyrolysis 2015, 112, 56-65. [CrossRef]

(C) 2020 by the authors. Licensee MDPI, Basel, Switzerland. This article is an open access article distributed under the terms and conditions of the Creative Commons Attribution (CC BY) license (http://creativecommons.org/licenses/by/4.0/). 